\title{
Sistema Experto Probabilístico basado en Redes Bayesianas para la predicción del cáncer de cuello uterino
}

\section{Probabilistic Expert System based on Bayesian Networks for the prediction of cervical cancer}

\author{
Luis A. Paulino Flores *, Ana M. Huayna Dueñas \\ Universidad Nacional Mayor de San Marcos, Facultad de Ingeniería de Sistemas e Informática. Lima, Perú \\ *Email: alonsopf1@gmail.com
}

\begin{abstract}
Resumen
El cáncer de cuello uterino es el cuarto cáncer más frecuente en la mujer. Una gran variedad de técnicas utilizadas en la Inteligencia Artificial (IA) como las Redes Neuronales, las Máquinas de Vectores de Soporte (SVM), los Árboles de Decisión y otros; han abordado el problema de la predicción de esta enfermedad. El siguiente artículo muestra la predicción de riesgo de cáncer de cuello uterino usando un modelo probabilístico basado en Redes Bayesianas; donde de un total de 322 registros se pudo obtener 15 atributos o características diferentes que correspondan a la información de una paciente. Las pruebas fueron realizadas utilizando el $40 \%$ de los datos, matrices de confusión y el indicador AUC. Los resultados le otorgan al trabajo desarrollado una tasa de éxito del $96 \%$ así como un valor de 0.9864 en términos del indicador AUC, además, sugieren que las Redes Bayesianas alcanzan un alto rendimiento, así como también ofrecen transparencia durante el proceso de inferencia, algo que no sucede con muchas otras técnicas, y que son ideales para afrontar problemas de predicción.
\end{abstract}

Palabras clave: Modelos Predictivos; Método de Bayes; Algoritmos de Predicción; Computación Probabilística.

\begin{abstract}
Cervical cancer is currently the fourth most frequent type of cancer in women. A large number of techniques from the Artificial Intelligence (AI) such as Neuronal Networks, Support Vector Machines (SVM), Decision Trees and others; have been used to deal with the problem of predicting this disease. The following paper shows the cervical cancer risk prediction, by implementing a probabilistic model based on Bayesian Networks and using 322 instances where we could retrieve 15 different features that are known information from each patient. The tests were made using the $40 \%$ of the whole dataset, confusion matrix and AUC indicator. The results show that this work has raised a $96 \%$ of success rate as well as 0.9864 in terms of the AUC indicator, in addition to this, the results suggest that Bayesian Networks are able to reach a high performance and provide transparency during the inference process at the same time, something that does not happen in many other techniques, and that they are really efficient to face this sort of prediction problems.
\end{abstract}

Keywords: Predictive models; Bayes methods; Prediction algorithms; Probabilistic Computing.

Correspondencia:

Dirección: Universidad Nacional Mayor de San Marcos, Facultad de Ingeniería de Sistemas e Informática. Calle Germán Amézaga № 375 , Ciudad Lima 1.

Recibido 23/04/2019 - Aceptado 30/05/2019

Citar como:

Paulino, L. \& Huayna, A. (2019) Sistema Experto Probabilístico basado en Redes Bayesianas para la predicción del cáncer de cuello uterino. Revista Peruana de Computación y Sistemas, 2(1):15-26. http://dx.doi.org/10.15381/rpcs.v2i1.16360

(C) Los autores. Este artículo es publicado por la Revista Peruana de Computación y Sistemas de la Facultad de Ingeniería de Sistemas e Informáticade la Universidad Nacional Mayor de San Marcos. Este es un artículo de acceso abierto, distribuido bajo los términos de la licencia Creative Commons Atribucion - No Comercia_Compartir Igual 4.0 Internacional. (http://creativecommons.org/licenses/by-nc-sa/4.0/) que permite el uso no comercial, distribución y reproducción en cualquier medio, siempre que la obra original sea debidamente citada. 


\section{Introducción}

El trabajo realizado durante las últimas décadas por investigadores procedentes de varios campos de la Inteligencia Artificial muestra cómo muchos de los problemas que antes parecían imposibles, o intratables, pueden hoy ser formulados y resueltos por máquinas [1].

El desarrollo de sistemas de diagnóstico basados en técnicas bayesianas comenzó en los años 60 . Tal y como señala F.J. Díez [2], estos primeros sistemas utilizaban el método probabilístico clásico, que, a pesar de presentar deficiencias, habría paso a futuras implementaciones, técnicas y mejoras basadas en probabilidades como lo son hoy en día las Redes Bayesianas, un Modelo Gráfico Probabilista (MGP) que sería visto por primera vez en los años 80 y a partir de allí, pasaría a ser la base de muchísimos sistemas expertos probabilísticos.

Según F.J. Díez [2], los MGP encajan perfectamente con la medicina, dado que esta última posee dos propiedades importantes: el conocimiento causal y las fuentes de incertidumbre. Precisamente el presente trabajo de investigación aborda la predicción de riesgo de cáncer de cuello uterino con la ayuda de un MGP, específicamente, las Redes Bayesianas.

El diagnóstico para el cáncer de cuello uterino requiere de una biopsia que debe ser tomada de una lesión cervical visible [3]. Existen diferentes procedimientos para llevarlo a cabo siendo el Papanicolaou uno de los más conocidos y que Organización Mundial de la Salud (OMS) recomienda realizar a las mujeres mayores de treinta años con cierta frecuencia. Según la OMS [4], en los países desarrollados, se han puesto en marcha programas que han logrado prevenir hasta el $80 \%$ de los casos de cáncer de cuello uterino, sin embargo, esto es algo que aún no se logra en los países en desarrollo. El Centro para el Control y Prevención de Enfermedades (CDC) menciona en [5] que, si bien ha habido una mejora significativa en reducir la tasa de mortalidad de la enfermedad, el progreso se ha visto estancado (Ver Figura 1), principalmente por falta de prevención y desinformación de muchas mujeres que no toman las medidas apropiadas o no son conscientes del riesgo que tienen de desarrollar esta enfermedad.
Las estadísticas y los números hablan por sí mismos acerca de la realidad de esta enfermedad alrededor del mundo. En Europa se estima que, durante el 2018, hubo 69000 nuevos casos de cáncer de cuello uterino y 30000 muertes a causa de la misma enfermedad según la OMS [28].

La realidad en África es incluso peor. En el sur de África, se reportan 43 casos de cáncer de cuello uterino por cada 100000 mujeres anualmente, comparado con los 6.8 por cada 100000 en Europa Occidental. La tasa de mortalidad es la mayor en el este de África, reportándose 30 muertes por cada 100000 mujeres mientras que en Europa Occidental el promedio de muertes es 2.1 por cada 100000 mujeres. Según la OMS, sin una intervención efectiva, las muertes a causa de cáncer de cuello uterino se duplicarían para el 2030, siendo el 90\% de estas en el continente africano [29].

Según el Instituto Catalán de Oncología (ICO), en Asia, cada año 315346 mujeres son diagnosticadas con cáncer de cuello uterino y 168411 mueren a causa de la misma enfermedad. Actualmente, es considerada la tercera enfermedad más frecuente en las mujeres de Asia [30].

Cada año más de 56000 mujeres en la región de Latinoamérica y el Caribe son diagnosticadas con cáncer cervical y más de 28000 mueren por la enfermedad. Incluyendo a Estados Unidos y Canadá, los números incrementan a 72000 y 34000 respectivamente. Según Silvana Luciani: "Es inaceptable que las mujeres hoy en día mueran a causa de una enfermedad que puede ser prevenida." [31].

Solamente en el Perú, al año se registran 4103 nuevos casos de cáncer de cuello uterino, de los cuales 1836 mujeres fallecen. La falta de cultura preventiva y temor de las mujeres a realizarse un chequeo preventivo hace que el $85 \%$ de casos sean detectados en una etapa avanzada de la enfermedad [32].

En el presente paper, presentamos un modelo probabilístico basado en Redes Bayesianas, con el objetivo de contribuir a la prevención y detección temprana de esta enfermedad con una herramienta capaz de hacerlo de forma automática, y construida en base al conocimiento de expertos en el área de Ginecología.

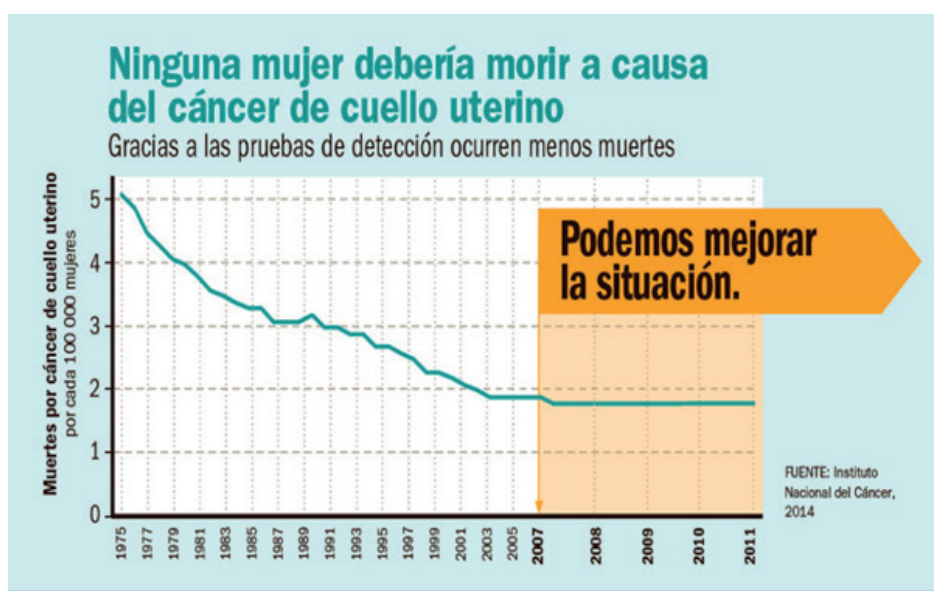

Figura 1. Tasa de mortalidad del cáncer de cuello uterino [5] 
Nuestra propuesta fue puesta a prueba realizando utilizando un total de 129 casos reales. Los resultados le otorgan al trabajo desarrollado una tasa de éxito del 96\% así como un valor de 0.9864 en términos del indicador AUC, además, sugieren que las Redes Bayesianas alcanzan un alto rendimiento, así como también ofrecen transparencia durante el proceso de inferencia, algo que no sucede con muchas otras técnicas, y que son ideales para afrontar problemas de predicción.

\section{Revisión Literaria}

En el año 2009 se presentaron dos casos de estudio relacionados al cáncer de cuello uterino, uno de ellos en Indonesia, donde el Departamento de Ingeniería Biomédica de la Universidad Swiss German [6] presentó un sistema para la detección temprana de cáncer de cuello uterino utilizando reconocimiento de patrones sobre imágenes citológicas de la zona cervical en 2D, con el fin de lidiar con el problema de inconsistencia e imprecisión del diagnóstico del test del Papanicolaou. Por otro lado, Onísko, Druzdzel y Marshall [7] implementaron un modelo basado en Redes Bayesianas Dinámicas al cual dieron el nombre de PCCSM (Pittsburgh Cervical Cancer Screening Model), el cual permite evaluar el riesgo de cáncer cervical invasivo en el transcurso del tiempo, con la finalidad de obtener una serie de resultados por paciente en diferente etapas cronológicas, lo cual ayudaría a los médicos a realizar un seguimiento individual a cada uno de ellos para tomar decisiones más certeras al momento de programar las evaluaciones médicas respectivas.

En el año 2012, nuevamente se presentaron dos casos de estudios muy interesantes. El primero fue desarrollado en Perú, por la alumna de pregrado, Barturen Sánchez [8], de la escuela de Ingeniería de Sistemas de la Universidad Católica Santo Toribio de Mogrovejo. El trabajo de investigación consistió en el desarrollo de un Sistema Experto basado en reglas de inferencia para el diagnóstico temprano de cáncer de cuello uterino. A pesar de contar con una cantidad de datos relativamente pequeñas (113 historias clínicas), el sistema experto logró alcanzar un grado de confianza del $97 \%$, un número bastante alto y muy alentador. Mientras tanto, Ricardo Timarán Pereira y María Clara Yépez Chamorro [9] desarrollaron en conjunto con la Universidad de Nariño en Colombia, un estudio cuya finalidad fue descubrir patrones de supervivencia en mujeres con cáncer invasivo de cuello uterino. Para llevar el proyecto a cabo se vieron en la necesidad de utilizar diferentes técnicas de clasificación y asociación basadas en árboles de decisión, siendo capaces de generar modelos bastante consistentes con la realidad observada. Dentro de sus principales resultados, se llegó a la conclusión que el $95.4 \%$ de mujeres muertas a causa del cáncer de cuello uterino cumplían con 3 características: no ser madre cabeza de familia, no estar clasificada en el SISBEN (Sistema de Selección de Beneficiarios para Programas Sociales) y no pertenecer a algún régimen de salud.

$\mathrm{Al}$ año siguiente, 2013, dos nuevos casos de estudio se dieron a conocer. La Revista Internacional de
Machine Learning y Computación (IJMLC por sus siglas en inglés) [10] publicó un estudio enfocado en el problema de la recurrencia del cáncer de cuello uterino en pacientes que alguna vez fueron diagnosticados con esta enfermedad. Haciendo uso de un conjunto de datos correspondiente a las historias clínicas de 168 pacientes fueron capaces de desarrollar dos modelos diferentes, ambos con técnicas diferentes, uno de ellos utilizó el algoritmo C5.0 mientras el otro utilizó el algoritmo de clasificación MARS. Los resultados demostraron que la efectividad de los árboles de decisión + C5.0 alcanzan un $96 \%$ de nivel de confianza superando al modelo de MARS que alcanzó un 86\%. De la misma manera, en la Conferencia Internacional de Computación Gráfica, Visualización y Visión Computacional [11] se presentó un estudio acerca de la identificación de regiones anormales en la zona cervical haciendo uso de imágenes extraídas del examen de colposcopía. Las pruebas realizadas sobre un conjunto de 48 imágenes pertenecientes a 12 pacientes con diferentes diagnósticos respecto al cáncer cervical, demostraron que, las Máquinas de Vectores de Soporte (SVM por sus siglas en inglés) alcanzan una tasa de éxito que asciende al $94.6 \%$ haciendo uso de una función Kernel lineal, que a pesar de ser la más simple, se comportó muchísimo mejor que las funciones de base radial y Polinomial.

En el año 2014, la Revista Internacional de Aplicaciones Computaciones (IJCA por sus siglas en inglés) [12] presentó un estudio realizado en Chennai, India, en el cual se logró implementar un modelo de detección temprana de cáncer haciendo uso de técnicas de Minería de Datos, específicamente de los famosos Árboles de decisión y de la técnica de Clustering: K-Means. Haciendo uso de un total de 746 casos de prueba, el 99\% fue clasificado correctamente. En el mismo año, en la conferencia MobiHealth [13], se dio a conocer un sistema basado en Redes Bayesianas cuyo objetivo era predecir el diagnóstico de pacientes que fueron sometidos a pruebas como el Papanicolaou y obtuvieron un resultado dudoso o ambiguo. Este sistema resultó bastante prometedor alcanzando un porcentaje de $94.9 \%$ en términos de Valor Predictivo Positivo y $95.5 \%$ en términos de Valor Predictivo Negativo (PPV y PPN por sus siglas en inglés, respectivamente).

Dos años más tarde, en el 2016, en Calicut, India, el Departamento de Ciencias de la Computación e Ingeniería de la Universidad Akkikavu [14] presentó una investigación basada en la detección y clasificación de cáncer cervical analizando la textura de imágenes extraídas del examen de Resonancia Magnética (MRI por sus siglas en inglés). Haciendo uso de modelos de clasificación basados en SVM no lineales y de los atributos recolectados de una serie de transformaciones y algoritmos aplicadas a las imágenes, como la transformación de Contourlet, o las características de Gabor, se concluyó que las imágenes obtenidas por MRI de tipo Sagital Ponderada T2 favorecen al buen desempeño del modelo alcanzando una precisión del 83\%, ligeramente superior a resultados obtenidos con imágenes Axiales 
Ponderadas T1 y T2 que obtuvieron $81 \%$ y $82 \%$ respectivamente. Por otro lado, también en la India, la Universidad de Manonmaniam Sundaranar [15] realizó una comparación entre distintos métodos aplicados para la predicción del cáncer de cuello uterino utilizando datos genéticos. Un total de 100 registros fueron utilizados en el proyecto, de los cuáles 60 correspondieron al conjunto de datos de entrenamiento y 40 al conjunto de pruebas. Se pusieron a prueba 3 técnicas diferentes: el algoritmo de CART, con el cual se alcanzó un 83.7\% de tasa de éxito; el algoritmo RFT, cuya tasa de éxito asciende a 93.54\%; y finalmente, un modelo que combina dos algoritmos: el RFT y el K-Means. Este último modelo obtuvo resultados muchísimo mejores que en los experimentos anteriores, logrando alcanzar una tasa de éxito de $96.77 \%$. Mientras tanto en Irán, la Universidad de Ciencias Médicas [16] publicó un estudio en el cuál reunió evidencia de diferentes publicaciones realizadas entre el 2005 y 2015 que demuestran que la aplicación de Redes Bayesianas para la predicción de enfermedades obtiene resultados altísimos comparado con otros algoritmos. (Ver Figura. 2).

En el ańo siguiente, 2017, la revista de Reconocimiento de Patrones [17] presentó un proyecto basado en clasificación de imágenes haciendo uso de Redes Neuronales Convolucionales (CNN por sus siglas en inglés) con la finalidad de mejorar la precisión del proceso de clasificación de displasias cervicales. Haciendo uso de un conjunto de 1112 imágenes de la zona cervical, cada una correspondiente a pacientes diferentes lograron desarrollar un método efectivo que alcanzó superar por un margen de $10 \%$ a muchos otros métodos incluyendo a conocidas pruebas como el Papanicolaou o las pruebas de HPV. De la misma manera, en China, la Universidad Politécnica de Anhui [18] publicó un artículo describiendo los resultados de una investigación acerca de la identificación de cáncer cervical utilizando imágenes de la zona cervical, principalmente basándose en la textura y en las áreas que presentan lesiones. El método utilizado combina diferentes técnicas enmarcadas en el campo de la Inteligencia Artificial, tales como el K-Means que fue utilizado para la segmentación y agrupación de la zona cervical siendo capaces de así, distinguir las áreas afectadas, y por último se utilizaron SVN para reconocer si en efecto, la zona analizada se trata de cáncer cervical o no. Los resultados experimentales muestran que la precisión alcanzada por el método propuesto, asciende a un $90 \%$ que, si bien es una tasa bastante alta, los autores mencionan que sería ideal comprobarlo con un número más alto de datos de prueba, dado que para los resultados expuestos únicamente se utilizaron 20 datos de prueba.

En el 2018, en Japón, el Departamento de Ginecología del Centro de Cáncer de Saitama [19] presentó un proyecto de investigación que pretende demostrar el uso de Deep Learning para abordar problemas de clasificación, en este caso en particular, la clasificación de imágenes obtenidas por el examen de colposcopía. Lamentablemente, los resultados no fueron los esperados, dado que la evaluación en términos de precisión alcan- zó el 50\% a diferencia de casos de estudio previamente analizados, sin embargo, los autores mencionan que es complicado comparar estos resultados con trabajos previamente implementados dado que es la primera vez que se decide aplicar Deep Learning para abordar el tema de clasificación de imágenes de colposcopía y que los resultados son lo suficientemente buenos a nivel clínico considerando la dificultad de los propios médicos para diferenciar entre diferentes displasias, incluso siendo ellos los expertos. En el mismo año, la Revista del Instituto Nacional de Cáncer [20] publicó un estudio que desarrolló un algoritmo capaz de automatizar el diagnóstico de displasias cervicales. Los resultados fueron bastante alentadores, siendo que, el algoritmo remitió al 91.7\% de los casos de HPV positivo CIN3/AIS a un examen de colposcopía inmediata, mientras que difirió un total del $38.4 \%$ de todas las mujeres con HPV positivo a una reevaluación de un año, lo cual, comparado con un examen directo de citología cuyos resultados eran de $89.1 \%$ y $37.4 \%$ respectivamente, lo convierten en, sin duda, un algoritmo confiable para el proceso de diagnóstico o triaje como es mencionado por los autores, de una displasia cervical. Por otro lado, en Los Ángeles, California, la Universidad del Sur de California realizó un estudio que fue publicado en la Revista Americana de Obstetricia y Ginecología [21] el cual consiste en la comparación de dos modelos diferentes, uno basado en el modelo de riesgo proporcional de Cox, y otro basado en Deep Learning para analizar las probabilidades de supervivencia de pacientes diagnosticados con cáncer de cuello uterino. Los resultados ofrecidos sugieren que el modelo basado en Deep Learning es una herramienta confiable para predecir la supervivencia de mujeres con cáncer de cuello uterino basándose en el error absoluto obtenido por cada uno de los diferentes modelos, 43.6\% con el modelo de Cox, y 30.7 con el modelo de Deep Learning, lo cual significa que su tasa de éxito ascendía al $69.3 \%$, pero eso no es todo, sino que, al incluir más características, del total de 40 que se tenían por paciente, los resultados mejoraban alcanzando una tasa de éxito de 79.5\% haciendo uso de las 40 .

Un año después, en el 2019, en la Revista del Instituto Nacional de Cáncer [22] se publicó un artículo que trata sobre la aplicación de Deep Learning y la evaluación automatizada de imágenes de la zona cervical para detectar cáncer de cuello uterino. Haciendo uso del enfoque de Deep Learning conocido como Faster R-CNN, se entrenó un modelo utilizando cervigramas digitalizados. El algoritmo en mención alcanzó un alto nivel de precisión, cuyo valor asciende al 91\%, superando de esta manera incluso a la interpretación original de los cervigramas que contaban con un $69 \%$ o a la evaluación citología convencional, que contaba con un 71\%. Asimismo, en Pakistán, 2 universidades de la ciudad de Lahore [23] publicaron un artículo en la Revista Internacional de Ciencias de la Computación Avanzada y Aplicaciones comparando diferentes métodos para predecir el cáncer de cuello uterino haciendo uso de Minería de Datos. El estudio presenta una tabla muy interesante (Ver Figura 3) donde se recopila diferentes 


\begin{tabular}{llllll}
\hline $\begin{array}{l}\text { Reference \& } \\
\text { Year }\end{array}$ & $\begin{array}{l}\text { Subject } \\
\text { Study }\end{array}$ & Illness & $\begin{array}{l}\text { Number } \\
\text { Of Variables }\end{array}$ & $\begin{array}{l}\text { Performance mea- } \\
\text { sure of NBN } \\
\text { (p-value00.01) }\end{array}$ & comparison \\
\hline $\begin{array}{l}\text { (2014) [5] } \\
\text { 35,605 patients } \\
\text { with lung cancer }\end{array}$ & $\begin{array}{l}\text { Brain metastasis } \\
\text { from } \\
\text { lung cancer }\end{array}$ & 6 Variables & $\begin{array}{l}\text { accuracy:82.83\% } \\
\text { Sensitivity:80.84\% } \\
\text { specificity:84.59\% }\end{array}$ & $\begin{array}{l}\text { BN:accuracy:82\% Sensitivity:83.28\% } \\
\text { specificity:80\% }\end{array}$ \\
\hline
\end{tabular}

\begin{tabular}{|c|c|c|c|c|c|}
\hline (2008) [11] & $\begin{array}{l}\text { Data of } 142 \text { brain } \\
\text { tumor patients }\end{array}$ & brain tumor & 96 attributes & $\begin{array}{l}\text { Accuracy: } 84 \% \\
\text { Specificity: } 87 \% \\
\text { Sensitivity: } 80 \%\end{array}$ & $\begin{array}{l}\text { BN:accuracy:80\% Sensitivity: } 73 \% \\
\text { specificity: } 85 \%\end{array}$ \\
\hline
\end{tabular}

\begin{tabular}{|c|c|c|c|c|c|}
\hline (2011) [20] & 1700 patients & $\begin{array}{l}\text { Prostate } \\
\text { Cancer }\end{array}$ & 4 variables & $A U C=66.2 \%$ & $\begin{array}{l}\text { TAN: } \\
\text { AUC }=58 \%\end{array}$ \\
\hline (2011) [9] & 3866 patient & $\begin{array}{l}\text { Minor } \\
\text { head } \\
\text { trauma }\end{array}$ & 17 attributes & $\begin{array}{l}\text { Sensitivity: } 95 \% \text { Spec- } \\
\text { ificity: } 95 \% \\
\text { AUC: } 95 \%\end{array}$ & $\begin{array}{l}\text { PIPPER: } \\
\text { Sensitivity: } 75.5 \% \text { Specificity: } 76.9 \% \\
\text { AUC: } 84.1 \%\end{array}$ \\
\hline (2013) [22] & 2318 patients & $\begin{array}{l}\text { glaucoma se- } \\
\text { verity }\end{array}$ & 6 variables & $\begin{array}{l}\text { Accuracy values } \\
\text { greater than } 80 \%\end{array}$ & (n.................. \\
\hline (2007) [17] & $\begin{array}{l}210 \text { high-risk } \\
\text { women }\end{array}$ & Breast Cancer & 6 features & $\begin{array}{l}\text { the ROC curve (AUC) } \\
: 67.5 \%\end{array}$ & -.........- \\
\hline (2013) [23] & $\begin{array}{l}119 \text { Chinese pa- } \\
\text { tients with (DKD) \& } \\
554 \text { without DKD }\end{array}$ & $\begin{array}{l}\text { diabetic } \\
\text { kidney disease }\end{array}$ & $\begin{array}{l}10 \text { clinical attri- } \\
\text { butes }\end{array}$ & Accuracy: $84 \%$ & $\begin{array}{l}\text { Partial least squares regression (PLS): } \\
\text { Accuracy: } 71 \%\end{array}$ \\
\hline (2010) [24] & 830 patients & $\begin{array}{l}\text { dialysis in ill pa- } \\
\text { tients }\end{array}$ & 2 input variables & AUC: $85.5 \%$ & SVM: AUC: $83.3 \%$ \\
\hline (2008) [16] & 128 patients & $\mathrm{BC}$ & 13 variables & $\begin{array}{l}\text { AUC : } 88.4 \% \\
\text { accuracy: } 81.8 \% \\
\text { sensitivity: } 75 \% \\
\text { specificity \& } \\
\text { PPV: between } 83 \% \\
\text { and } 86 \%\end{array}$ & $\begin{array}{l}\text { Logistic Regression (LR) :AUC : } 79.4 \% \\
\text { accuracy: } 76.1 \% \\
\text { sensitivity: } 75 \% \\
\text { specificity: } 83 \%\end{array}$ \\
\hline (2007) [25] & 169 patients & $\begin{array}{l}\text { acute appendi- } \\
\text { citis }\end{array}$ & 9 Variables & $\begin{array}{l}\text { ROC analysis showed: } \\
\text { BN model provided } \\
\text { the most reliable \& } \\
\text { accurate results. }\end{array}$ & $\begin{array}{l}\text { NBN works better than LR \& artificial } \\
\text { neural network (ANN) }\end{array}$ \\
\hline (2013) [26] & 240 patients & AE. & 42 Variables & Accuracy: $68 \%$, & $\begin{array}{l}\text { Decision Tree (DT): } \\
\text { Accuracy: } 64.1 \%\end{array}$ \\
\hline (2011) [10] & 1411 patients & $A D$. & $\begin{array}{l}312318 \text { (single nu- } \\
\text { cleotide polymor- } \\
\text { phisms } \\
(\text { SNPs)) SNP }\end{array}$ & AUC: $59 \%$ & $\begin{array}{l}\text { LR: } \\
\text { AUC: } 61.3 \%\end{array}$ \\
\hline (2012) [27] & 40 patients & Toothache & $\begin{array}{l}14 \text { pain parameters } \\
(P=14) .\end{array}$ & Accuracy :72\% & -......................... \\
\hline (2010) [28] & $\begin{array}{l}\text { population of } 558 \\
\text { Italian SSc. }\end{array}$ & SSc. & $\begin{array}{l}19 \\
\text { Variable }\end{array}$ & $\begin{array}{l}\text { accuracy: } 76.9 \% \text { sen- } \\
\text { sitivity: } 72.2 \% \text { speci- } \\
\text { ficity: } 81.6 \%\end{array}$ & $\begin{array}{l}\text { LR: accuracy: } 75.5 \% \text { sensitivity: } 69.4 \% \\
\text { specificity: } 81.6 \%\end{array}$ \\
\hline (2006) [29] & 1086 patients & anesthesia & $\begin{array}{l}11 \text { Preoperative and } \\
\text { intraoperative char- } \\
\text { acteristics }\end{array}$ & $\begin{array}{l}\text { AUC: } 57 \% \\
\text { accuracy: } 77 \% \text {; sen- } \\
\text { sitivity : } 18.3 \% \text {, spec- } \\
\text { ificity: } 95.7 \% \text {, PPV: } \\
57.6 \% \\
\text { NPV: } 78.6 \% \text {. }\end{array}$ & $\begin{array}{l}\text { LR: ROC curve: } 66.9 \% \\
\text { accuracy: } 64.2 \% \text {; sensitivity : } 62.5 \% \text {, } \\
\text { specificity: } 64.7 \% \text {, PPV: } 36.1 \% \\
\text { NPV: } 84.4 \% \text {. }\end{array}$ \\
\hline
\end{tabular}

\begin{tabular}{|c|c|c|c|c|c|}
\hline (2013) [30] & 583 patients & LV & $\begin{array}{l}12 \\
\text { Attributes }\end{array}$ & $\begin{array}{l}\text { accuracy: } 82.16 \% \\
\text { sensitivity }: 82.35 \% \\
\text { specificity: } 83 \% \text {, }\end{array}$ & $\begin{array}{l}\text { NN: accuracy: } 79 \% \text {; sensitivity : } 77.54 \% \text {, } \\
\text { specificity: } 75.83 \% \text {, }\end{array}$ \\
\hline (2007) [31] & 59 patients & AE. & 5 Variables & $\begin{array}{l}\text { Sensitivity: } 85 \% \\
\text { Specificity: } 78 \% \text { Ac- } \\
\text { curacy: } 82 \% \\
\text { AUC: } 88 \%\end{array}$ & DT: Sensitivity: $79 \%$ Specificity: $94 \%$ \\
\hline (2012) [32] & 987 patients & CAD & 113 Variables & AUC: $78 \%$ & SVM: AUC: $75 \%$ \\
\hline (2013) [33] & $\begin{array}{l}26 \text { adult asthma } \\
\text { patients }\end{array}$ & AE. & 20 Variables & $\begin{array}{l}\text { Sensitivity: } 80 \% \\
\text { Specificity: } 77 \% \text { Ac- } \\
\text { curacy: } 77 \%\end{array}$ & $\begin{array}{l}\text { SVM: } \\
\text { Sensitivity: } 84 \% \\
\text { Specificity: } 80 \% \text { Accuracy: } 80 \%\end{array}$ \\
\hline (2013) [34] & 45 subjects & $\begin{array}{l}\text { dementia in Par- } \\
\text { kinson's disease }\end{array}$ & 4 Variables & $\begin{array}{l}\text { Sensitivity: } 92.33 \% \\
\text { Specificity: } 100 \% \text { Ac- } \\
\text { curacy: } 93.33 \%\end{array}$ & $\begin{array}{l}\text { Filter Selection NB: } \\
\text { (FSNB) } \\
\text { Sensitivity: } 86 \% \text { Specificity: } 100 \% \text { Accu- } \\
\text { racy: } 93.33 \%\end{array}$ \\
\hline (2015) [35] & $\begin{array}{l}345 \text { type } 2 \\
\text { diabetic patients }\end{array}$ & $\begin{array}{l}\text { type } 2 \\
\text { diabetic }\end{array}$ & 7 features & $\begin{array}{l}\text { Sensitivity: } 41.03 \% \\
\text { Specificity: } 84.96 \% \\
\text { Accuracy: } 62.61 \%\end{array}$ & $\begin{array}{l}\text { SVM: } \\
\text { Sensitivity: } 50 \% \text { Specificity: } 78.72 \% \text { Ac- } \\
\text { curacy: } 60.87 \%\end{array}$ \\
\hline (2007) [36] & 2,949 subjects & CAD & 11 variables & $\begin{array}{l}\text { Sensitivity: } 88.3 \% \\
\text { Specificity: } 88.6 \% \text { Ac- } \\
\text { curacy: } 88.4 \%\end{array}$ & $\begin{array}{l}\text { NN (MLP) } \\
\text { Sensitivity: } 87.1 \% \text { Specificity: } 85.3 \% \text { Ac- } \\
\text { curacy: } 86.2 \%\end{array}$ \\
\hline (2012) [37] & $\begin{array}{l}227 \text { healthy new- } \\
\text { born infants }\end{array}$ & $\begin{array}{l}\text { neonatal jaun- } \\
\text { dice }\end{array}$ & 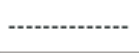 & AUC: $88 \%$ & $\begin{array}{l}\text { Bayes Net: } \\
\text { AUC: } 87 \%\end{array}$ \\
\hline
\end{tabular}

Figura 2. Evidencia recolectada por la Universidad de Ciencias Médicas sobre la efectividad de las Redes Bayesianas para predecir enfermedades. [16]. 
trabajos relacionados al cáncer cervical con diferentes métodos enmarcados en la Inteligencia Artificial, en ella se puede apreciar los resultados en función de precisión y/o curva de ROC.

El estudio además utilizó tres técnicas diferentes de la Minería de Datos para compararlas entre sí, las cuales fueron: Árboles de Decisión, Bosque de Decisión y Jungla de Decisión. Los resultados fueron que, en todos los experimentos, los Árboles de Decisión superaron a las otras 2 técnicas mencionadas. Se realizaron 4 experimentos tomando 4 variables diferentes del conjunto de datos como objetivo, cuando la variable biopsia era la variable objetivo, los Árboles de decisión alcanzaron un 97.4\% de precisión, cuando la variable citología fue considerada como la variable objetiva, la precisión alcanzó el 95.9\%, para la variable Test de Schiller como objetivo, se obtuvo 94.3\% y finalmente para la variable Test de Hinselmann, la precisión ascendió a $97.8 \%$, todos resultados bastante alentadores a favor de los Árboles de Decisión.

\section{Metodología}

La metodología consiste de tres pasos principales; el primero es la selección del conjunto de datos, seguido del tratamiento de datos que consiste en asegurarnos que el conjunto de datos esté listo para ser utilizado y finalmente la construcción del modelo probabilístico.

\subsection{Selección del Conjunto de Datos}

El conjunto de datos utilizados corresponde al repositorio público UCI [24] que cubre características y registros médicos históricos de un conjunto total de 858 personas. Se incluye una cantidad significativa de pacientes que decidieron no responder alguna de las preguntas por un tema de privacidad. La validez del conjunto de datos fue revisada por expertos en el área de Ginecología del Hospital de la Fuerza Aérea del Perú (FAP).

\begin{tabular}{|c|c|c|c|c|c|}
\hline \multirow{2}{*}{ Reference } & \multicolumn{3}{|l|}{ Data Set } & \multirow{2}{*}{ Technique } & \multirow{2}{*}{ Results } \\
\hline & Repository & Attributes & Instances & & \\
\hline$[20]$ & Universitario de Caracas Hospital patients & 28 & 858 & Hybrid method using deep learning & $\mathrm{AUC}=0.6875$ \\
\hline [24] & NCBI & 61 & 160 & CART Algorithm & Accuracy $=83.87 \%$ \\
\hline \multirow{3}{*}{ [25] } & \multirow{3}{*}{$\begin{array}{l}\text { Chung Shan Medical University Hospital } \\
\text { Tumor Registry }\end{array}$} & \multirow{3}{*}{38} & \multirow{3}{*}{75} & Naïve Bayes & Accuracy $=78.93 \%$ \\
\hline & & & & SVM & Accuracy $=78.67 \%$ \\
\hline & & & & Random Forest Tree & Accuracy $=80.18 \%$ \\
\hline [21] & $\begin{array}{l}\text { Bucheon St Mary's Hospital, Republic of } \\
\text { Korea }\end{array}$ & 15 & 731 & SVM & Accuracy $=74.41 \%$ \\
\hline [17] & $\begin{array}{l}\text { Chung Shan Medical University Hospital } \\
\text { Tumor Registry }\end{array}$ & 12 & 168 & MARS & Accuracy $=86.00 \%$ \\
\hline \multirow{4}{*}{ [18] } & \multirow{4}{*}{ State Hospital in Rzeszow } & \multirow{4}{*}{10} & \multirow{4}{*}{107} & GEP & AUROC $=0.72$ \\
\hline & & & & MLP & AUROC $=0.67$ \\
\hline & & & & PNN & AUROC $=0.56$ \\
\hline & & & & RBFNN & AUROC $=0.48$ \\
\hline [26] & Universitario de Caracas Hospital patients & 18 & 858 & $\begin{array}{l}\text { Transfer Learning with Partial } \\
\text { observability }\end{array}$ & RMSE $=35.11$ \\
\hline \multirow{7}{*}{ [27] } & \multirow{7}{*}{$\begin{array}{l}\text { Clinical data from patients treated surgically } \\
\text { in 1998-2001. }\end{array}$} & \multirow{7}{*}{23} & \multirow{7}{*}{102} & PNN & AUROC $=0.818$ \\
\hline & & & & MLP & AUROC $=0.659$ \\
\hline & & & & GEP & AUROC $=0.651$ \\
\hline & & & & SVM & AUROC $=0.478$ \\
\hline & & & & LRA & AUROC $=0.559$ \\
\hline & & & & RBFNN & AUROC $=0.640$ \\
\hline & & & & k-Means & AUROC $=0.406$ \\
\hline
\end{tabular}

Figura 3. Evidencia recolectada por las Universidades de Ingeniería y Tecnología, y de Gestión y Tecnología de Lahore, Pakistán [23]. 


\subsection{Tratamiento de Datos}

Uno de los puntos más importantes para comenzar y llegar a implementar un modelo que requiere alimentarse de datos, es el de garantizar que el conjunto de datos es el adecuado para la técnica que se va a aplicar. Garantizar que el conjunto de datos está en buena forma significa que las probabilidades de que el experimento sea real y verosímil son bastante altas. Para este caso de estudio, se procedió analizando el conjunto completo de 858 instancias extraídas del repositorio UCI y eliminar aquellos registros que presentaban la mayor cantidad de atributos faltantes. Dado que uno de nuestros objetivos es hacer que nuestro modelo aprenda de un conjunto de datos, es importante que el conjunto del cual va a aprender sea lo más completo posible, un paciente del cuál no se conocen ni siquiera la mitad de las variables no añade mucho valor al proceso de aprendizaje del modelo probabilístico.

Como resultado nos quedamos con 322 registros en total para proseguir con la implementación del modelo basado en Redes Bayesianas.

La cantidad total de atributos en el conjunto de datos original es de 36, sin embargo, muchos de estos atributos también tienen valores faltantes, lo que significa que un gran número personas no recuerda o simplemente no desea revelar. Así como también hay atributos que están tan fuertemente relacionados que pueden ser inclusive tratados como uno solo, o cuya relevancia está inmediatamente siendo considerada dentro de otra variable, lo cual nos hace prescindir del mismo.

Todo ello nos conlleva a limpiar el conjunto de datos de forma de quedarnos únicamente con aquello que sea necesario, relevante y se adapte a la metodología. Luego de haber realizado un análisis y prescindir de algunos atributos, el conjunto de datos final utilizado contiene un total de 322 registros y 15 variables cada uno (Ver Tabla 1), incluyendo la variable binaria target, la cual indica si el paciente fue diagnosticado con cáncer de cuello uterino o no.

Muchas de las variables son de tipo continuo, lo que significa que hace falta un proceso de Discretización de Variables, el cual consiste en convertir todas las variables continuas en variables discretas haciendo uso de estados (valores discretos) que representen intervalos en los que cada variable continua pueda ser ubicada. Por ejemplo, la variable Edad es una variable continua que necesita ser discretizada, para ello, los valores de la variable Edad se dividen en 4 estados, cada uno de ellos representando un intervalo, el primero de todas las personas cuya edad está entre 15 y 25 inclusive, el segundo de todas las personas cuya edad está entre 26 y 36 inclusive, el tercero entre 36 y 46 inclusive y finalmente el cuarto formado por todas las personas cuya edad es igual o mayor a 46.

La realidad es que existe una gran cantidad de técnicas y algoritmos dentro del marco de la Inteligencia Artificial que permiten abordar una gama diferente de problemas. La razón por la cual el presente trabajo utiliza y aplica el concepto de Redes Bayesianas es porque se han identificado una serie de ventajas y puntos fuertes que esta técnica en particular tiene y se acomoda perfectamente a lo que se pretende alcanzar. Para poder evaluar y comparar las Redes Bayesianas con otras técnicas se identificaron una serie de criterios o características (Ver Tabla 2) donde se les asignó un puntaje.

Tabla 1

Variables utilizadas para la creación de la Red.

\begin{tabular}{ll} 
Variable & Tipo \\
Edad & Continuo \\
Edad primera relación sexual & Continuo \\
\# Parejas Sexuales & Continuo \\
\# Embarazos & Continuo \\
ETS & Discreto \\
\# ETS & Continuo \\
DIU & Discreto \\
\# Años con DIU & Continuo \\
Anticonceptivos Hormonales & Discreto \\
\# Años con anticonceptivos hormonales & Continuo \\
Fuma & Discreto \\
\# Años Fumando & Continuo \\
Test de Schiller & Discreto \\
Colposcopía & Discreto \\
Target & Discreto \\
\hline
\end{tabular}

\subsection{Construcción del Modelo Probabilístico}

Luego, se procedió a desarrollar un Benchmarking (Ver Tabla 3) de las diferentes técnicas mencionadas en la revisión de la literatura, donde las Redes Bayesianas, y los Árboles de Decisión + RFT alcanzan el puntaje más alto entre todas las técnicas consideradas. Los Árboles de Decisión + RFT alcanzan un puntaje bajísimo en el atributo de Número de Datos, mientras que las Redes Bayesianas obtienen la mejor puntuación. Además, las Redes Bayesianas no necesitan de un conjunto de entrada completo, sino que es posible utilizar como entrada incluso una sola variable de todo el conjunto usado en la red. Al conjunto de entrada que utiliza las Redes Bayesianas se le llama evidencia, que es, en otras palabras, aquellas variables que se puede garantizar que pueden asumir un valor en específico con una probabilidad igual a 1. Es por estas razones que se opta por el uso de las Redes Bayesianas para el presente caso de estudio.

Para proceder con la construcción del modelo se utilizó la ayuda del Software Netica 6.0.5. Netica es un software desarrollado por la empresa Norsys, y es una herramienta poderosa y fácil de utilizar al momento de trabajar con Redes Bayesianas. Netica ofrece una interfaz bastante intuitiva que permite al usuario interactuar con la Red Bayesiana gráficamente, así como también tiene muchos de los algoritmos de aprendizaje ya implementados, como el algoritmo EM y otros más como el Counting-Learning. 
Tabla 2

Atributos a considerar para evaluación de las diferentes técnicas de la Inteligencia Artificial.

\begin{tabular}{|c|c|c|c|}
\hline ID & ATRIBUTO & DESCRIPCIÓN & PUNTOS \\
\hline \multirow{2}{*}{ A } & \multirow{2}{*}{ Naturaleza de datos } & El método sólo admite que el dato de entrada sea discreto o que sea variable. & 1 \\
\hline & & El método es adaptable a ambos tipos de datos. & 2 \\
\hline \multirow{2}{*}{ B } & \multirow{2}{*}{$\begin{array}{l}\text { Cantidad de datos de } \\
\text { entrenamiento }\end{array}$} & No se requiere de muchos datos de entrenamiento para alcanzar un alto rendimiento. & 2 \\
\hline & & Se requiere de un alto número de datos de entrenamiento para alcanzar un alto rendimiento. & 1 \\
\hline \multirow{3}{*}{$\mathrm{C}$} & \multirow{3}{*}{ Interpretabilidad } & El método no cuenta con un razonamiento simbólico y representación semántica. & 0 \\
\hline & & El método es descriptivo y requiere cierta interpretación. & 1 \\
\hline & & Se presentan los resultados de manera visual o al menos de manera que su interpretación sea muy clara. & 2 \\
\hline \multirow{2}{*}{$\mathrm{D}$} & \multirow{2}{*}{ Velocidad } & $\begin{array}{l}\text { El método requiere de un alto costo computacional, incluso si la cantidad de datos a manipular no es } \\
\text { alta, por lo que los tiempos de respuesta son lentos. }\end{array}$ & 1 \\
\hline & & $\begin{array}{l}\text { El método no siempre requiere de un alto costo computacional, dependiendo de la cantidad de } \\
\text { datos a manipular, los tiempos de respuesta pueden ser incluso inmediatos. }\end{array}$ & 2 \\
\hline \multirow{3}{*}{$\mathrm{E}$} & \multirow{3}{*}{ Precisión } & El método tiene una precisión $<=70 \%$. & 1 \\
\hline & & La precisión está entre $70 \%$ y $85 \%$. & 2 \\
\hline & & El método tiene un indicador de precisión mayor a $85 \%$ & 3 \\
\hline \multirow{3}{*}{$\mathrm{F}$} & \multirow{3}{*}{ Manejo de ruido } & El método tiende a cometer grandes imprecisiones cuando recibe datos con alta presencia de ruido. & 1 \\
\hline & & $\begin{array}{l}\text { Significa que la técnica es capaz de reconocer algunos datos ruidosos, sin embargo, no es constante y } \\
\text { su tasa de precisión puede verse afectada notablemente. }\end{array}$ & 2 \\
\hline & & $\begin{array}{l}\text { Se maneja eficientemente cualquier dato recibido, almacenado o editado sin que esto afecte en gran } \\
\text { medida la precisión del resultado. }\end{array}$ & 3 \\
\hline \multirow{3}{*}{ G } & \multirow{3}{*}{ Área de dominio } & La construcción no requiere ningún dominio de conocimiento. & 3 \\
\hline & & Se requiere una delimitación en el dominio de trabajo para la construcción. & 2 \\
\hline & & Se necesita conocimiento completo del dominio que abarca el sistema. & 1 \\
\hline \multirow{3}{*}{$\mathrm{H}$} & \multirow{3}{*}{ Nivel de complejidad } & $\begin{array}{l}\text { Los algoritmos utilizados son sencillos e intuitivos, con una curva de aprendizaje alta, y no requieren } \\
\text { experiencia previa para ser implementados. }\end{array}$ & 3 \\
\hline & & $\begin{array}{l}\text { La implementación de los algoritmos tienen una curva media de aprendizaje, no son complicados de } \\
\text { implementar, pero si requieren experiencia previa para su buen entendimiento. }\end{array}$ & 2 \\
\hline & & $\begin{array}{l}\text { El método utiliza algoritmos complejos que requieren de una alta curva de aprendizaje y son difíciles } \\
\text { de implementar si no se cuenta con experiencia previa. }\end{array}$ & 1 \\
\hline
\end{tabular}

Tabla 3

Benchmarking de diferentes técnicas utilizadas dentro de la Inteligencia Artificial.

\begin{tabular}{lccccccccc}
\hline Técnicas vs Atributos & A & B & C & D & E & F & G & H & Total \\
\hline SVM & 2 & 1 & 1 & 1 & 3 & 3 & 3 & 1 & 15 \\
CART & 2 & 1 & 2 & 1 & 2 & 2 & 3 & 2 & 15 \\
RFT & 2 & 1 & 2 & 1 & 3 & 3 & 3 & 2 & 17 \\
C5.0 & 2 & 1 & 2 & 1 & 3 & 3 & 3 & 2 & 16 \\
ID3 & 2 & 1 & 2 & 1 & 2 & 2 & 3 & 1 & 14 \\
Redes Bayesianas & 2 & 2 & 2 & 2 & 2 & 3 & 2 & 2 & 17 \\
Reglas de Inferencia & 1 & 2 & 2 & 2 & 2 & 1 & 1 & 3 & 14 \\
Redes Neuronales & 1 & 1 & 0 & 2 & 3 & 3 & 3 & 2 & 15 \\
Algoritmos Genéticos & 1 & 2 & 1 & 2 & 2 & 2 & 1 & 1 & 12 \\
K-Means & 2 & 1 & 1 & 2 & 3 & 1 & 2 & 3 & 15 \\
KNN & 2 & 1 & 1 & 1 & 3 & 2 & 2 & 3 & 15 \\
\hline
\end{tabular}

Como ya se mencionó, la topología y estructura de la Red Bayesiana fue desarrollada de forma manual con el apoyo de expertos en el dominio que enmarca al cáncer de cuello uterino, es por ello que, con la ayuda de Netica, se diagramó la topología del modelo probabilístico (Ver Figura 4).

Una se definió la estructura y topología de la Red Bayesiana, tuvo lugar el aprendizaje paramétrico.
Nuevamente con la ayuda de Netica 6.0.5 se ejecutó el algoritmo EM para un conjunto total de 193 registros, los cuales representan el $60 \%$ del conjunto total de datos (322). La consola de Netica muestra el progreso y cada una de las iteraciones del algoritmo EM durante la fase de aprendizaje, para este caso de estudio fueron necesarias únicamente tres iteraciones para que el algoritmo EM detecte que ya no existen mayores cambios significativos respecto a las iteracio- 
nes previas. Se muestra en la Figura 5 que, durante la segunda iteración, las probabilidades variaron hasta en un $47.6519 \%$, sin embargo, con los ajustes hechos durante las dos fases del algoritmo EM en la siguiente iteración, los cambios fueron reducidos a $0.0 \%$ por lo que el proceso de aprendizaje se considera finalizado (Ver Figura 5).

Luego de haber ejecutado este proceso se puede ver como Netica asigna una serie de valores a cada nodo de la Red Bayesiana (Ver Figura 6). Estos valores representan las probabilidades marginales y condicionales utili- zadas durante el proceso del algoritmo EM, dependiendo de si son nodos raíz o no respectivamente.

Luego de la Estimación Paramétrica, se puede observar que cada nodo tiene una tabla de probabilidad asociada. Las tablas de probabilidad ayudan a inferir la probabilidad de una variable no observada basándose en la evidencia o valores previamente inferidos de los nodos padres. Netica, por supuesto, también ofrece una interfaz bastante elegante para trabajar con las tablas de probabilidades. La forma como las tablas están organizadas es la siguiente: la tabla se divide en dos secciones grandes, la

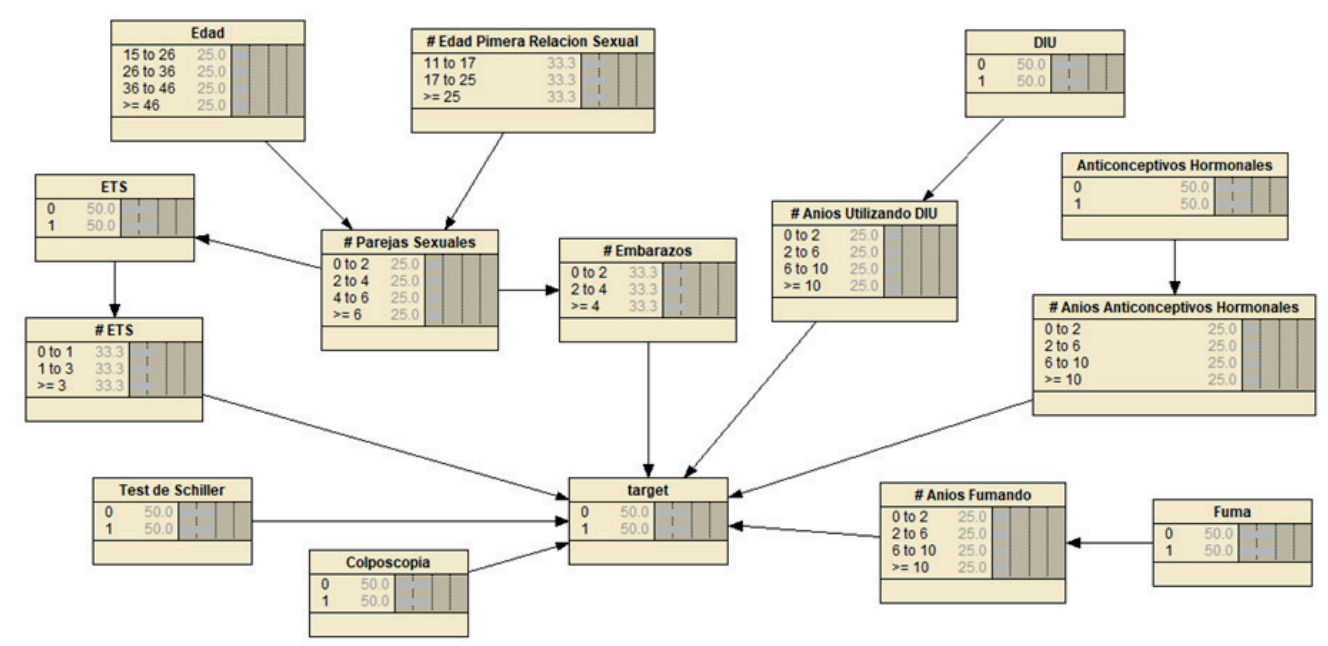

Figura 4. Topología del modelo probabilístico

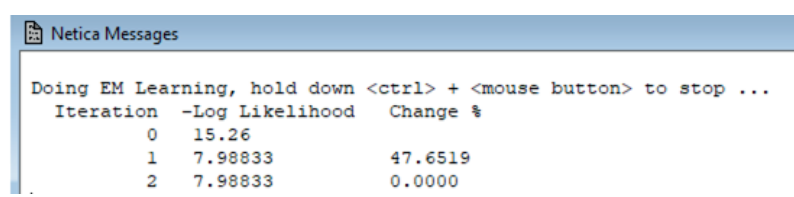

Figura 5. Consola de Netica durante la fase de aprendizaje de la Red Bayesiana.

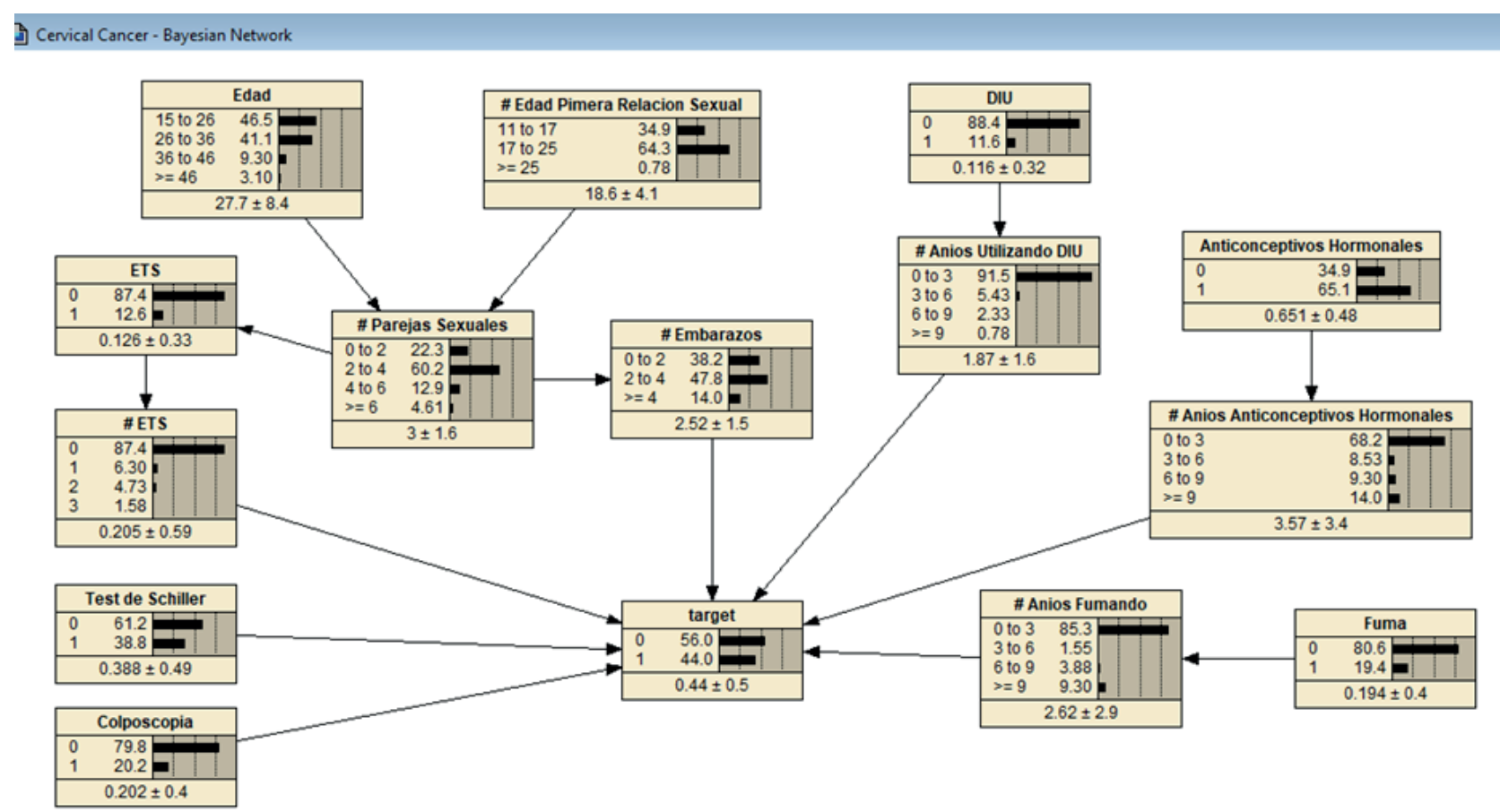

Figura 6. Estado de los nodos de la Red Bayesiana luego del Aprendizaje Paramétrico 
sección izquierda, es sub dividida en columnas, una por cada nodo padre del nodo en análisis, mientras tanto, la sección derecha es dividida a su vez en columnas, una por cada valor que la variable del nodo en análisis puede tomar. Luego, en cada una de las celdas de la sección de la izquierda se colocan todas las combinaciones posibles para los diferentes valores que pueden tomar cada uno de las variables de los nodos padre, finalmente, cada una de estas combinaciones afectará a la probabilidad de cada uno de los diferentes estados o valores listados en la sección derecha (Ver Figura 7 y Figura 8).

\section{Resultados y Discusión}

Nuevamente con la ayuda de Netica, se hizo una evaluación de 129 casos (40\% del total de casos) para poder obtener métricas acerca del funcionamiento del modelo probabilístico propuesto. Para ello fue necesario primero, compilar la red, luego es necesario seleccionar una variable objetivo, para ello se utilizó la variable target que es justamente la que se necesita evaluar, pues contiene la información sobre el diagnóstico de cáncer de cuello uterino. Finalmente se procedió seleccionando el conjunto de 129 registros para poner a prueba la Red Bayesiana.

Con la ayuda de Netica se aplicó el algoritmo Belief Propagation para 129 registros de pacientes diferentes obteniéndose los resultados que se muestran en la Figura 9 y la Figura 10.

Tal y como se puede observar en la Figura 9, de un total de 55 personas diagnosticadas con cáncer de cuello uterino, 51 fueron evaluadas correctamente, mientras que de 74 personas cuyo resultado era negativo, se clasificó correctamente a 73. En otras palabras, de los 129 casos, solo 5 fueron mal clasificados, los cuales representan el $3.876 \%$ de margen de error y significa que la tasa de éxito del modelo alcanza el 96.124\% 96\%.

\begin{tabular}{|c|c|c|c|c|c|c|}
\hline \multicolumn{5}{|c|}{ A_Parejos__Seruales Table (in Bayes net Cervical_Cancer_Network) } & \multicolumn{2}{|c|}{$0 \times \sqrt{-x}$} \\
\hline \multicolumn{5}{|c|}{ Node:__Parejas_Sexuales $\quad \boldsymbol{\nabla}$} & \multirow{2}{*}{$\begin{array}{l}\text { Apply } \\
\text { Reset }\end{array}$} & \multirow{2}{*}{$\begin{array}{c}\text { OK } \\
\text { Close }\end{array}$} \\
\hline Chance & $\nabla$ & \% Probability $\nabla$ & & & & \\
\hline Edad & \multicolumn{2}{|c|}{ \# Edad Pimera Relacion Sexual } & 0 to 2 & 2 to 4 & 4 to 6 & $>=6$ \\
\hline 15 to 26 & \multicolumn{2}{|l|}{11 to 17} & 14.286 & 67.857 & 17.857 & $3.57 e-5$ \\
\hline 15 to 26 & \multicolumn{2}{|l|}{17 to 25} & 40.625 & 46.875 & 9.375 & 3.125 \\
\hline 15 to 26 & \multicolumn{2}{|l|}{$>=25$} & 25 & 25 & 25 & 25 \\
\hline 26 to 36 & \multicolumn{2}{|l|}{11 to 17} & 23.077 & 38.461 & 23.077 & 15.385 \\
\hline 26 to 36 & \multicolumn{2}{|l|}{17 to 25} & 10.256 & 76.923 & 7.692 & 5.128 \\
\hline 26 to 36 & \multicolumn{2}{|l|}{$>=25$} & 99.997 & $1.00 e-3$ & $1.00 \mathrm{e}-3$ & $1.00 e-3$ \\
\hline 36 to 46 & \multicolumn{2}{|l|}{11 to 17} & $3.33 \mathrm{e}-4$ & 99.999 & $3.33 \mathrm{e}-4$ & $3.33 \mathrm{e}-4$ \\
\hline 36 to 46 & \multicolumn{2}{|l|}{17 to 25} & 22.222 & 66.666 & 11.111 & $1.11 e-4$ \\
\hline 36 to 46 & \multicolumn{2}{|l|}{$>=25$} & 25 & 25 & 25 & 25 \\
\hline$>=46$ & \multicolumn{2}{|l|}{11 to 17} & $1.00 e-3$ & $1.00 e-3$ & 99.997 & $1.00 e-3$ \\
\hline$>=46$ & \multicolumn{2}{|l|}{17 to 25} & $3.33 e-4$ & 99.999 & $3.33 \mathrm{e}-4$ & $3.33 \mathrm{e}-4$ \\
\hline$>=46$ & \multicolumn{2}{|l|}{$>=25$} & 25 & 25 & 25 & 25 \\
\hline
\end{tabular}

Figura 7. Tabla de probabilidad para la variable \#Parejas Sexuales

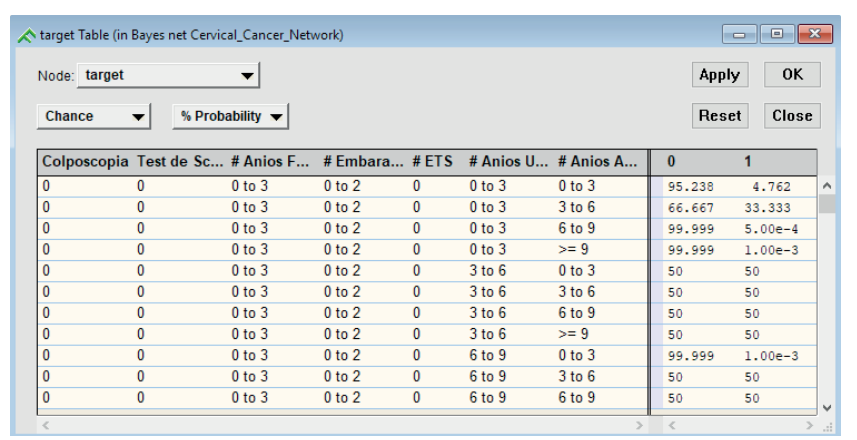

Figura 8. Extracto de la tabla de probabilidad para la variable target

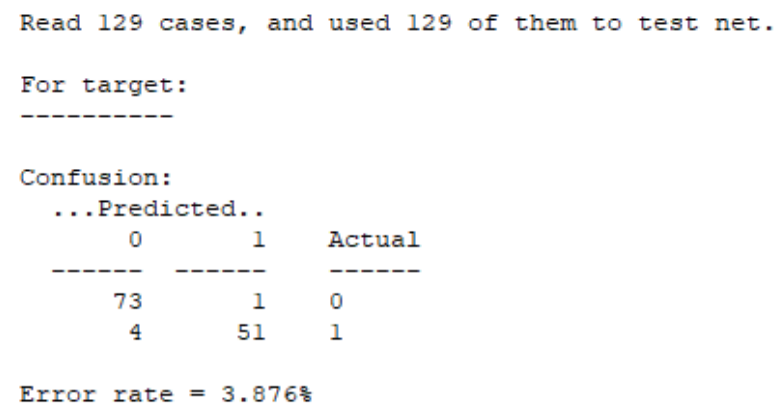

Figura 9. Matriz de Confusión de la Red Bayesiana 


$\begin{array}{crrrrr}\text { Quality of Test for state '(null)': } & & & \\ \text { Cutoff } & \text { Sensitivity } & \text { Specificity } & \text { Predictive } & \text { Predict-Neg } & \text { Error-Rate } \\ 0 & 100.00 & 0.00 & 57.36 & 100.00 & 42.64 \\ 0.001 & 100.00 & 85.45 & 90.24 & 100.00 & 6.20 \\ 98 & 39.19 & 100.00 & 100.00 & 55.00 & 34.88 \\ 100 & 0.00 & 100.00 & 100.00 & 42.64 & 57.36 \\ \text { Gini coeff }=0.9727 & & & & \\ \text { Area under ROC }=0.9864 & & & & \end{array}$

Figura 10. Coeficiente de Gini y Área debajo de la curva de ROC

Por otro lado, la Figura 10 muestra los cálculos que llevan a concluir en dos valores interesantes, el coeficiente de Gini y el área debajo de la curva ROC (AUC, por sus siglas en inglés). El primero es un valor que oscila entre - 1 y 1 , un valor alto del coeficiente de Gini, como el obtenido (0.9727), sugiere que el modelo es bueno y consistente. Lo mismo sucede con el coeficiente obtenido de la curva de ROC que oscila entre 0 y 1 . Un modelo cuyas predicciones son $100 \%$ incorrectas tiene AUC igual a 0 , mientras que un modelo cuyas predicciones son perfectas, tiene un AUC igual a 1. Dado que el valor del coeficiente AUC obtenido en la prueba es igual a 0.9864 , altamente cercano a 1 , podemos concluir que el modelo es confiable y tiene un alto rendimiento.

Los resultados obtenidos por el modelo probabilístico basado en Redes Bayesianas del presente trabajo de investigación lograron superar largamente, en términos de precisión, a todos los experimentos realizados entre el año 2005 y 2015 con una cantidad de atributos y número de registros similares que fueron mencionados por la Universidad de Ciencias Médicas, Irán (ver figura 2).

Uno de los trabajos más recientes, publicado en la Revista Internacional de Ciencias de la Computación Avanzada mencionaba una lista de trabajos previamente implementados en donde no fueron utilizadas las Redes Bayesianas. Haciendo una pequeña comparación entre los resultados obtenidos en esta investigación con los resultados de la investigación desarrollada por dos universidades de Pakistán mostrados en la figura 3, se puede ver que las Redes Bayesianas superan a todos los métodos mencionados en términos de precisión y AUC, lo cual es un muy buen indicador e incentiva a seguir aplicando esta técnica a diferentes casos de estudios relacionados con predicción.

\section{Conclusiones}

Se implementó un modelo probabilístico basado en Redes Bayesianas capaz de clasificar con una tasa de éxito de $96 \%$ a personas diagnosticadas con cáncer de cuello uterino. Además de ello, gracias a la versatilidad y transparencia que ofrecen las Redes Bayesianas, se puede analizar las probabilidades de cada una de las variables. Las Redes Bayesianas has demostrado poder alcanzar un rendimiento bastante alto ofreciendo total transparencia sobre el proceso de inferencia, algo que no pasa con muchas otras técnicas que por lo general ofrecen un resultado, pero sin tener en cuenta la incertidumbre existente y más aún, sin poder explicar el cómo se llega a esa conclusión. Si bien es cierto el objetivo principal era el de desarrollar un modelo confiable capaz de predecir el riesgo de desarrollar cáncer cervical, esta pequeña característica de visibilidad y transparencia de las Redes Bayesianas abren las puertas a muchísimos análisis con cada una de las variables envueltas apoyadas cada una en su propia tabla de probabilidades.

A pesar de los muy buenos resultados, no hay que dejar de recordar que el conjunto de datos utilizado fue de 322 instancias y 15 atributos. Sería ideal poder realizar más experimentos con esta técnica sobre conjuntos de datos muchísimo más grandes, que incluyan miles de instancias y muchos más atributos. Hoy en día el principal obstáculo es la recolección de datos, ya que se requiere de bastante tiempo, paciencia, perseverancia $y$, sobre todo, colaboración por parte de las instituciones de salud para proveer la mayor cantidad de datos posibles de las pacientes atendidas para fines académicos.

\section{Referencias}

[1] E. Castillo, J. M. Gutiérrez, y A. S. Hadi, Expert Systems and Probabilistic Network Models. Springer-Verlag, New York, 1997.

[2] F. J. Díez. Aplicaciones de los modelos gráficos probabilistas en medicina. En: J. A. Gámez y J. M. Puerta (eds.), Sistemas Expertos Probabilísticos, págs. 239-263. Universidad de Castilla-La Mancha, Cuenca, 1998.

[3] Guía de práctica clínica de cáncer de cuello uterino. Dpto de Oncología Médica, Instituto Nacional de Enfermedades Neoplásicas, Perú, 2013.

[4] Papilomavirus humanos (PVH) y cáncer cervicouterino', https://www.who.int/es/news-room/fact-sheets/detail/human-papillomavirus-(hpv)-and-cervical-cancer, accessed 5 February 2019.

[5] 'Millones de mujeres en los EEUU no se están haciendo las pruebas de detección de cáncer de cuello uterino.' Atlanta (GA): Centers for Disease Control and Prevention; 2014. https://www.cdc.gov/spanish/mediosdecomunicacion/comunicados/p_vs_cancer_cuello_uterino_110514.html, accessed November 262016.

[6] Suryatenggara J, Ane BK, Pandjaitan M, Steinberg W. Pattern recognition on $2 \mathrm{D}$ cervical cytological digital images for early detection of cervix cancer. Proceedings of the World Congress on Nature and Biologically Inspired Computing (NABIC '09); December 2009; Coimbatore, India. pp. 257-262.

[7] Onísko, A., Druzdzel, M. J., \& Austin, R. M. (2009). Application of Dynamic Bayesian Networks to Cervical Cancer Screening. Intelligent Information Systems 9999, 1-10.

[8] Barturen S., Lourdes. (2012). Desarrollo de un sistema experto sobre web para un diagnóstico temprano de cáncer de 
cuello uterino en la Clínica Maternidad "Belen" - Chiclayo. Escuela de Ingeniería de Sistemas y Computación, Universidad Católica Santo Toribio de Mogrovejo, Lambayeque, Perú.

[9] Timarán R, Yépez M. La minería de datos aplicada al descubrimiento de patrones de supervivencia en mujeres con cáncer invasivo de cuello uterino, Universidad y salud, 117-129, 2012.

[10] Chang, C.-C., Cheng, S.-L., Lu, C.-J., \& Liao, K.-H. (2013) Prediction of Recurrence in Patients with Cervical Cancer Using MARS and Classification. International Journal of Machine Learning and Computing vol. 3, no. 1, 75-78.

[11] Liang, M., Zheng, G., Huang, X., Milledge, G., \& Tokuta, A. (2013). Identification of Abnormal Cervical Regions from Colposcopy Image Sequences. 21st International Conference in Central Europe on Computer Graphics, Visualization and Computer Vision, 130-136.

[12] Ramachandran, P., Girija, N., \& Bhuvaneswari, T. (2014). Early Detection and Prevention of Cancer using Data Mining Techniques. International Journal of Computer Applications, 97(13), 48-53.

[13] Bountris, P., Tsirmpas, C., Koutsouris, D., \& Haritou, M. (2014). Bayesian Networks to Support the Management of Patients with ASCUS/LSIL Pap Tests. Conference: 4th International Conference on Wireless Mobile Communication and Healthcare (MOBIHEALTH), 103 - 107.

[14] Soumya, M., Sneha, K., \& Arunvinodh, C. (2016). Cervical Cancer Detection and Classification using Texture Analysis. Biomedical and Pharmacology Journal, 9(2), 663 - 671.

[15] R.Vidya and G. M. Nasira, "Prediction of Cervical Cancer using Hybrid Induction Technique: A Solution for Human Hereditary Disease Patterns", Indian Journal of Science and Technology, vol 9(30), 2016.

[16] Langarizadeh M, Moghbeli F. Applying naive bayesian networks to disease prediction: a systematic review. Acta Inform Med. 2016;24(5):364.

[17] T. Xu, H. Zhang, C. Xin, E. Kim, L. R. Long, Z. Xue, S. Antani, and $X$. Huang, "Multi-feature based benchmark for cervical dysplasia 11 classification evaluation," Pattern Recognition, vol. 63 , pp. 468-475, 2017.

[18] Lisheng Wei, Quan Gan \& Tao Ji (2017) Cervical cancer histology image identification method based on texture and lesion area features, Computer Assisted Surgery, 22:sup1, 186-199, DOI: 10.1080/24699322.2017.1389397.

[19] Sato, M., Horie, K., Hara, A. et al, Application of deep learning to the classification of images from colposcopy. Oncol Lett. 2018; 15:3518-3523.

[20] Kai Yu, Noorie Hyun, Barbara Fetterman, A. et al, Automated Cervical Screening and Triage, Based on HPV Testing and Computer-Interpreted Cytology (2018). Journal of the National Cancer Institute. 110(10), 1222-1228.
[21] Matsuo, Koji \& Purushotham, Sanjay \& Jiang, Bo \& S. Mandelbaum, Rachel \& Takiuchi, Tsuyoshi \& Liu, Yan \& Roman, Lynda. (2018). Survival outcome prediction in cervical cancer: Cox models versus deep-learning model. American Journal of Obstetrics and Gynecology. 10.1016/j.ajog.2018.12.030.

[22] Liming Hu, David Bell, Sameer Antani, Zhiyun Xue, Kai Yu, A. et al. (2019) An Observational Study of Deep Learning and Automated Evaluation of Cervical Images for Cancer Screening, JNCl: Journal of the National Cancer Institute, djy225, https://doi.org/10.1093/jnci/djy225.

[23] Mahboob, Talha \& Milhan, Muhammad \& Iqbal, Muahammad \& Wahab, Abdul \& Mushtaq, Mubbashar. (2019). Cervical Cancer Prediction through Different Screening Methods using Data Mining. International Journal of Advanced Computer Science and Applications. 10. 388-396

[24] Dua, D. and Graff, C. (2019). UCl Machine Learning Repository [http://archive.ics.uci.edu/ml]. Irvine, CA: University of California, School of Information and Computer Science.

[25] Suárez I., Mario. Probabilidad total y Teorema de Bayes, https://www.monografias.com/trabajos89/probabilidad-total-y-teorema-bayes/probabilidad-total-y-teorema-bayes.sht$\mathrm{ml}$, accessed 18 August 2018

[26] Carvalho, Pedro. Bayesian Networks. (2015, Dec. 09). https://rationalistramble.wordpress.com/2015/12/09/bayesian-networks/, accessed 10 July 2017.

[27] Luis Enrique Sucar. Redes Bayesianas. INAOE. Sta. María Tonatzintla, Puebla, 72840, México. https://ccc.inaoep.mx/ esucar/Clases-mgp/caprb.pdf, accessed 10 January 2019.

[28] 'Word Cancer Day: action for protection against cervical cancer',http://www.euro.who.int/en/health-topics/noncommunicable-diseases/cancer/news/news/2019/2/world-cancerday-action-for-protection-against-cervical-cancer, accessed 3 May 2019

[29] 'HIV and cervical cancer - a perfect storm for women in Africa',https://www.avert.org/news/hiv-and-cervical-cancer\%E2\%80\%93-perfect-storm-women-africa, accessed 3 May 2019

[30] ICO/IARC HPV Information Centre. Human Paillomavirus and Related Diseases Report. Version posted at www.hpvcenter.net on 10 December 2018

[31] 'Cervical cancer is the third most common cancer among women in Latin America and the Caribbean, but it can be prevented', https://www.paho.org/hq/index. php?option $=$ com content $\&$ view $=$ article\&id $=14947$ : cer vical-cancer-is-the-third-most-common-cancer-amongwomen-in-latin-america-and-the-caribbean-but-it-can-beprevented\&ltemid=1926\&lang=en, accessed 3 May 2019

[32] 'Conoce los 4 métodos para prevenir el cáncer de cuello uterino'. https://peru21.pe/vida/conoce-4-metodos-prevenir-cancer-cuello-uterino-nndc-467853, accessed 5 May 2019. 